\title{
Long pulse delays in thin metamaterial slabs
}

\author{
Nikitas Papasimakis, Vassili A. Fedotov, Nikolay I. Zheludev \\ Optoelectronics Research Centre, University of Southampton, Southampton, SO17 1BJ, UK \\ Sergey L. Prosvirnin \\ Institute of Radio Astronomy, National Academy of Sciences of Ukraine, Kharkov, 61002, Ukraine
}

We provide experimental evidence that an electromagnetic pulse can be significantly delayed with minimum loss and distortion, while propagating through a new type of planar meta-materials that supports modes weakly coupled to free-space radiation ("dark modes"). Although changes in the velocity and shape of light pulses propagating through bulk dispersive media have been studied extensively in the past [1], these effects have recently drawn considerable attention, since the ability to accurately control the velocity of light is essential for the development of optical communication technologies. We show that the pulse velocity can be controlled through appropriate subwavelength patterning of a thin metal film, namely a planar metamaterial. As typical examples of such structures, we consider two metamaterial arrays with a $15 \times 15 \mathrm{~mm}$ unit cell lithographically etched on a $1.5 \mathrm{~mm}$ thick PCB dielectric substrate. The first structure is a bi-layered "fish-scale" [2] consisting of an array of continuous wavy metallic strips residing on both faces of the substrate (see inset to Fig. 1a), while the second is an array of split ring resonators with asymmetric arc lengths (see inset to Fig. 1b). As it can be seen from the experimental spectra of Fig. 1a and Fig. 1b, both planar metamaterials exhibit strong normal dispersion in a transmission window $(\sim 5.5 \mathrm{GHz})$ as a result of "dark mode" excitation.

(a)

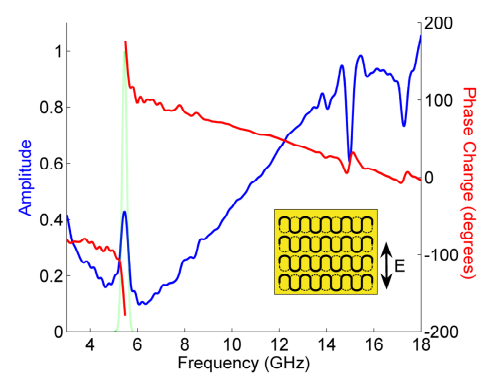

(b)

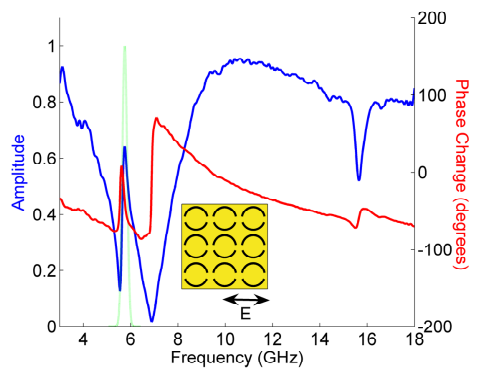

(c)

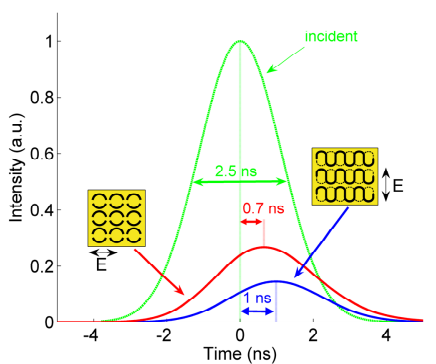

Fig. 1: (a) Transmission amplitude (blue), phase change (red) and frequency spectrum of incident pulse (dash green) for the bi-layered "fish-scale" shown in the inset (the pattern on the back face of the slab is represented by the dash lines in the inset). (b) Transmission amplitude (blue), phase change (red) and frequency spectrum of incident pulse (dash green) for the split ring array shown in the inset. (c) Response of the bi-layered "fish scale" (red) and the split ring array (blue) to a $2.5 \mathrm{~ns}$ long pulse (green).

The response of the metamaterials to microwave pulses can be reconstructed from the experimental spectrum by employing the inverse Fourier transform. To examine the stuctures' response to Gaussian-shaped pulses we consider 2.5 ns long pulses with their center frequency located in the transmission band of each structure. The frequency spectra of the incident pulses are shown in Figs $1 \mathrm{a} \& 1 \mathrm{~b}$, respectively. When the incoming pulse propagates through the planar metamaterial, it experiences a time delay of about 0.7-1 ns, which corresponds roughly to $\sim 0.3-0.4$ of the pulse width, while transmission reaches $25 \%$ (see Fig. 1c). Moreover, with the exception of a weak broadening, the pulses propagate with no distortion retaining their original Gaussian shape. This is remarkable in view of the vanishing thickness of the metamaterials (35 times thinner than the wavelength), which enables the successive stacking of metamaterial slabs in order to achieve very high time delays, however at the expense of pulse intensity. Furthermore, the use of geometrical resonances allows wide scalability and operation at prescribed wavelengths. Finally, we expect that such structures can find applications as ultra-compact delay lines and phase shifters.

\section{References}

1. L. Brillouin, Wave Propagation and Group Velocity (Academic Press Inc., New York, 1960).

2. V. A. Fedotov et al. "Planar electromagnetic metamaterial with a fish scale structure," Phys. Rev. E 72, 056613 (2005). 